\section{Associação entre distúrbios do ombro e trabalho: breve revisão da literatura}

\section{Association between shoulder diseases and work: a brief review}

Hélio Pires de Mendonça Jr. ${ }^{1 *}$

\section{Ada Ávila Assunção²}

'Departamento do Aparelho Locomotor, Faculdade de Medicina, Universidade Federal de Minas Gerais

2Programa de Pós-Graduação em Saúde Pública, Faculdade de Medicina, Universidade Federal de Minas Gerais

\section{Resumo}

Este artigo apresenta os resultados de uma breve revisão da literatura focalizada nos distúrbios do ombro. As publicações foram pesquisadas nos sites do Medline, Ovid, Lilacs e periódicos Capes, no período de janeiro de 1993 a julho de 2004, com as seguintes palavras-chave: shoulder pain, rotator cuff, job, dor no ombro, manguito rotador, trabalho. Foram selecionados os artigos de acordo com os seguintes critérios: estudo epidemiológico publicado em inglês, português ou espanhol, com informações sobre a carga física ou sobre os fatores psicossociais do trabalho, explicitação do método de avaliação da exposição, presença de dor no ombro ou tendinopatia do manguito rotador. Vinte artigos preencheram todos os critérios de inclusão. Encontrou-se associação positiva entre os distúrbios do ombro e o trabalho praticado pelos sujeitos dos estudos revisados. Restaram como limite da revisão: a heterogeneidade dos artigos quanto à avaliação da exposição, ao tipo de diagnóstico, à metodologia utilizada na condução da pesquisa, e aos meios para avaliação da dor. Os autores discutem a necessidade de aprofundamento dos métodos de investigação de problemas musculoesqueléticos relacionados ao trabalho.

Palavras-chave: Dor no Ombro. Manguito Rotador. Trabalho.

*Correspondência: Av. Professor Alfredo Balena, 190/ 8009, 30130-100 Belo Horizonte, MG.

E-mail: heliopiresmj@ig.com.br. 
Abstract

This article presents the results of a brief review of shoulder diseases and their association with work. Pertinent studies, identified by the keywords shoulder pain, rotator cuff, and job were reviewed in the reference databases Medline, Ovid, Lilacs and Capes journals (January 1993 to July 2004). The articles were chosen according to the following criteria: either epidemiological or experimental surveys having been published in English, Portuguese or Spanish, with information about physical demands or psychosocial factors of work, evaluation of exposure, presence of shoulder pain or rotator cuff lesion. After the search, 20 articles were included in the revision. The articles show a relationship between shoulder diseases and working in awkward postures. The limitations to the revision observed were: heterogeneity of the exposure and diagnosis, methodological problems and the methods to evaluate pain. The authors discuss the need of new methods of investigation for work-related musculoskeletal diseases.

Key Words: Shoulder Pain. Rotator Cuff. Work.

\section{Introdução}

O objetivo deste artigo é apresentar os resultados de uma breve revisão da literatura sobre distúrbios de ombro, buscando evidências de associação com o trabalho. $O$ estudo teve a sua origem em uma pesquisa anterior que evidenciou uma prevalência desses transtornos em uma população de metalúrgicos.

O ombro pode ser sede de uma variedade de lesões, a saber: estiramento, inflamação, fibroses, lesão incompleta ou completa do manguito rotador, associada ou não a degeneração articular. São várias as causas que concorrem para o desenvolvimento dos distúrbios do ombro, entre as quais o trauma, a hipovascularização na inserção do músculo supra-espinhoso e o impacto subacromial primário. A causa mais comum é o processo de envelhecimento. Os distúrbios de ombro são raros antes dos 40 anos e aumentam na faixa de 50 a 60 anos, continuando a crescer a partir dos 70 anos. A síndrome do manguito rotador tem sido descrita em associação com exposições a movimentos repetitivos de braço, elevação e abdução dos braços acima da altura dos ombros'.

À semelhança de outros países, no Brasil os dados oficiais sobre a prevalência de distúrbios músculo-esqueléticos não apresentam estratificação segundo a região acometida ou diagnóstico firmado. As estatísticas do Instituto Nacional de Seguridade Social (INSS) mostram aumento da concessão de benefícios por Distúrbios Osteomusculares ${ }^{2}$ Relacionados ao Trabalho (DORT). Segundo os dados disponíveis, respondem por mais de $80 \%$ dos diagnósticos que resultaram em concessão de auxílio-acidente e aposentadoria por invalidez pela Previdência Social, em 1998 ${ }^{3}$. Entre eles, encontram-se: capsulite adesiva, síndrome do manguito rotador, tendinite bicipital e tendinite calcárea do ombro.

O Departamento de Seguros da Suécia mostra que, em 1994, cerca de $18 \%$ do total de indenizações por ausência no trabalho foram devidas a problemas no ombro e no pescoço ${ }^{4}$. No Estado de Washington, no período de 1987 a 1995, houve diagnóstico de 55.315 casos de problemas de ombro relacionados ao traba- 
lho (média de 6.146 casos por ano), sendo que 2/3 desses trabalhadores ficaram afastados do trabalho mais de quatro dias, com média de 97 dias de afastamento. As indústrias relacionadas foram: construção civil, coleta de lixo e madeireiras. A categoria das enfermeiras registrou elevado número de casos ${ }^{5}$.

$\mathrm{Na}$ Espanha, é também encontrado um fenômeno parecido: os distúrbios músculoesqueléticos de origem ocupacional, que, em 1988, eram $30 \%$ do total de doenças ocupacionais, passaram a representar $76 \%$ daquele total, em $1997^{6}$.

Na Grã-Bretanha, estudo por meio de um questionário sobre distúrbios músculoesqueléticos de origem ocupacional estimou que, aproximadamente, 500.000 pessoas são acometidas por tenossinovites, epicondilite, síndrome do túnel do carpo. Já na Dinamarca, houve aumento no número de notificações desses distúrbios, de 3.576 casos, em 1993, para 4.168 casos, em $1998^{6}$.

Na Bélgica, 39\% de uma população estudada apresentaram queixas músculoesqueléticas de origem ocupacional, nos 12 meses anteriores à pesquisa. Já na França, estudo realizado, por meio de questionários enviados para os donos de empresa, demonstrou risco elevado de distúrbios músculo-esqueléticos na indústria da construção civil, agricultura e pesca ${ }^{6}$.

\section{Material e métodos}

As publicações foram pesquisadas nos sites Medline (janeiro de 1993 a julho de 2004), Ovid (janeiro de 1993 a julho de 2004), Lilacs (janeiro de 1993 a julho de 2004) e periódicos Capes (janeiro de 1993 a julho de 2004), com as seguintes palavras-chave: shoulder pain, rotator cuff, job, dor no ombro, manguito rotador, trabalho. Todas as publicações, no período de janeiro de 1993 a julho de 2004, foram selecionadas.

Como critérios de inclusão, os estudos escolhidos deveriam preencher as seguintes condições:

- estudo epidemiológico (série de casos, transversal, caso-controle ou coorte) ou experimental;
- publicado em inglês, português ou espanhol;

- com informações sobre a carga física do trabalho ou fatores psicossociais do trabalho;

- com a utilização de método padrão (observacional, entrevistas ou questionários) na avaliação da exposição;

- presença de dor no ombro ou tendinopatia do manguito rotador confirmados por meio de questionários, entrevistas, exame clínico ou testes laboratoriais (Tomografia Computadorizada ou Ressonância Magnética).

Foram excluídos os artigos sobre lesões agudas ou traumas ocorridos durante atividades esportivas e cartas à redação e editoriais .

Para cada artigo foram extraídos detalhes quanto à população de estudo, à exposição aos fatores de risco e ao acompanhamento. A força de associação foi avaliada baseada nos seguintes critérios: relação temporal, validade interna, força de associação e plausibilidade biológica da associação.

O projeto desta pesquisa foi aprovado pelo Comitê de Ética em Pesquisa da Universidade Federal de Minas Gerais.

\section{Fatores de risco ocupacionais e doença do manguito rotador}

A procura nos arquivos eletrônicos citados identificou 300 citações. Após a exclusão dos artigos utilizando-se dos critérios estabelecidos, 60 artigos foram selecionados para leitura, tendo sido incluídos 20 artigos, entre eles, a revisão do National Institute Occupational Safety and Health (NIOSH). Cinco artigos tinham desenho tipo transversal, três eram estudos tipo caso-controle, sete eram desenho tipo coorte e quatro eram experimentais (Tabela1).

Em vinte artigos, encontrou-se uma correlação entre dor no ombro e trabalho. A revisão do NIOSH mostrou-se bastante útil e citada na literatura em geral, uma vez que apresenta de maneira clara e sintética os principais resultados e métodos utilizados 
Tabela 1 - Estudos que pesquisaram a associação entre posições viciosas e carregamento de peso durante o trabalho com dor no ombro.

Table 1 - Studies that investigate the association between awkward postures and weight lifting during work and shoulder pain.

\begin{tabular}{|c|c|c|c|c|c|}
\hline Autor, ano & País & Desenho & Ocupações & Fatores de risco & Força de Associação \\
\hline Stenlund, 1993 & Suécia & Transversal & Construção civil & Carregamento de peso e vibração & OR: 2,$26 ;$ IC: $0,8-6,38$ \\
\hline Vasseljen, 1995 & Noruega & Caso controle & Trabalhadores de escritório & Uso de computadores & $p>0,001$ \\
\hline Sporrong, 1998 & Suécia & Experimental & & Elevação anterior e abdução & \\
\hline Fredriksson, 1999 & Suécia & Coorte & Empresas da Dinamarca & Período de descanso insatisfatórios & $\mathrm{RP}: 4,0$ \\
\hline Mäkelä, 1999 & Finlândia & Transversal & Empresas da Finlândia & Carga física elevada & OR: 2,$2 ;$ IC: $1,6-3,0$ \\
\hline Frost, 1999 & Dinamarca & Transversal & Trabalhadores de abatedouro & Elevação anterior do ombro & OR: 7,$9 ;$ IC: $2,94-21,18$ \\
\hline Punnett, 2000 & EUA & Caso controle & Indústria automobilística & Elevação anterior e abdução & OR: 4,0; IC:1,7-9,4 \\
\hline Kaergaard, 2000 & Dinamarca & Coorte & Operadoras de máquina de costura & Elevação anterior e abdução & OR: 2,26; IC: 1,1-5,9 \\
\hline Björklund, 2000 & Suécia & Experimental & & Elevação anterior e abdução & \\
\hline Palmerud, 2000 & Suécia & Experimental & & $\begin{array}{l}\text { Elevação anterior e abdução } \\
\text { Carga no membro }\end{array}$ & \\
\hline Pope, 2001 & Inglaterra & Transversal & $\begin{array}{l}\text { Construção civil. Enfermeiras, } \\
\text { Operadores de produção }\end{array}$ & $\begin{array}{l}\text { Elevação anterior e carregamento } \\
\text { de peso }\end{array}$ & $\mathrm{RP}: 2,6 ; \mathrm{IC}: 1,8-3,7$ \\
\hline Miranda, 2001 & Finlândia & Coorte & Trabalhadores florestais & $\begin{array}{l}\text { Elevação anterior e carregamento } \\
\text { de peso }\end{array}$ & OR:2,4; IC: 1,7-3,4 \\
\hline Feveile, 2002 & Dinamarca & Coorte & Empresas da Dinamarca & Elevação anterior e baixo suporte social & OR:1,5: IC 1,.1-2,06 \\
\hline Hoozemans, 2002 & Holanda & Coorte & Empresas da Holanda & Empurrar e puxar objetos & RR: 3,95; IC: 5,6-9,96 \\
\hline Fredriksson, 2002 & Suécia & Caso controle & Empresas da Suécia & Carregar peso e vibração & RR: 1,$9 ;$ IC: $0,6-5,5$ \\
\hline Cassou, 2002 & França & Coorte & Empresas da França & $\begin{array}{l}\text { Repetitividade, posturas viciosas e alta } \\
\text { demanda física }\end{array}$ & $\mathrm{P}<0,001$ \\
\hline Andersen, 2002 & Dinamarca & Transversal & Empresas da Dinamarca & Trabalho monótono e repetitividade & RP: 2,$3 ;$ IC: $1,4-4.0$ \\
\hline Stenlund, 2002 & Suécia & Experimental & Pintores de parede & Elevação anterior e abdução & \\
\hline Madeleine, 2003 & Dinamarca & Coorte & Trabalhadores de frigorífico & Elevação anterior & $P<0,001$ \\
\hline
\end{tabular}

em pesquisas publicadas no período de 1979 a 1996 que, em sua maioria, evidenciam associação entre exposição ocupacional e distúrbios de ombro?.

Para avaliar o efeito do trabalho sobre lesões do ombro, Stenlund et al. (1993) conduziram estudo transversal entre trabalhadores suecos de três setores da construção civil: pedreiros $(n=54)$, perfuradores de rocha $(n=55)$ e engenheiros $(n=98)$. A exposição foi avaliada baseada nos parâmetros: quantidade de peso carregada durante a jornada de trabalho, exposição à vibração e anos de trabalho manual. Os perfuradores foram classificados como tendo alta exposição, os pedreiros, como média exposição e os engenheiros, como baixa exposição do ombro a posturas viciosas durante o processo de trabalho. Todos eles foram então examina- dos por um ortopedista e um fisioterapeuta, para pesquisa de sintomas de tendinite no ombro. Os resultados mostraram que $40 \%$ dos perfuradores tinham sinais de tendinite no ombro direito e $33 \%$, apresentavam sinais de tendinite no ombro esquerdo. Entre os pedreiros e os engenheiros, $8 \%$ a $15 \%$ apresentavam sinais de tendinite no ombro. A exposição à vibração por longos períodos mostrou aumentar o risco para tendinite de ombro (OR: 1,66). O peso transportado ou o tempo de trabalho manual também foram associados ao aumento do risco de tendinite nessa região (OR: 2,26) ${ }^{8}$.

Estudo transversal, no período de 1987 a 1990, conduzido por Mäkelä et al. (1999), incluiu 8000 pessoas de todas as regiões e classes sociais da Finlândia, as quais foram entrevistadas com o auxílio de um questioná- 
rio e foram submetidas a exames laboratoriais e a testes funcionais para o ombro. Informações sobre fatores de risco foram colhidas por meio de questionário e de entrevista. Em 1998, foram reavaliadas 7042 pessoas, sendo novamente aplicado um questionário e realizado teste de função para os ombros. Nos resultados, encontrou-se prevalência de $8,8 \%$ de problemas nos ombros da população estudada. A análise univariada encontrou relação entre dor no ombro e baixo nível educacional, ocupação, estresse físico no trabalho (Odds Ratio de 2,2; IC 1,6-3,0), história pregressa de trauma no ombro, obesidade, diabetes e número de gestações. Os autores concluíram, então, que, devido ao desenho transversal do estudo, não seria possível fazer relação causal entre trabalho e problemas no ombro, entretanto, queixas nos ombros foram fortemente relacionadas ao passado de alto estresse físico no trabalho, sugerindo que atividade com alta demanda física pode causar problemas no ombro 9 .

Com o intuito de avaliar o risco de síndrome do impacto do ombro, Frost e Andersen (1999), na Dinamarca, realizaram estudo transversal em 1.141 trabalhadores de um abatedouro (casos) e de uma indústria química (controles). Para avaliar as queixas dos ombros, foram enviados questionários pelo correio aos participantes do estudo. Os pacientes com sintomas de longa duração nos ombros foram, então, selecionados para se submeterem a exame físico. Nos resultados, ficou evidente que os funcionários do abatedouro mantinham o braço elevado acima de $30^{\circ}$ por aproximadamente metade da jornada de trabalho, quando comparados com os trabalhadores da indústria química. As queixas de dor no ombro, assim como o exame positivo para síndrome do impacto, foi maior entre os funcionários do abatedouro do que entre os funcionários da indústria química (Odds Ratio de 7,90 com intervalo de confiança 2,94-21,18) ${ }^{10}$.

Pope et al. (2001) realizaram estudo transversal entre carteiros, caixas, operadores de produção e enfermeiras da GrãBretanha, objetivando avaliar a associação entre dor no ombro e a demanda física e psicossocial do trabalho. A todos os trabalhadores (775) foi entregue um questionário. Encontrou-se uma prevalência de $26 \%$ de queixas no ombro. Uma proporção elevada de trabalhadores antigos, comparados com trabalhadores novatos, apresentou dor no ombro. Trabalhar com flexão acima do nível do ombro e a duração do carregamento de peso foram associados com dor no ombro $^{11}$.

O estudo transversal, realizado por Andersen et al. (2002), numa população de 3.123 trabalhadores que executam atividades monótonas e repetitivas, de 19 companhias da Dinamarca, procurou avaliar os fatores de risco ocupacionais para dor no ombro e no pescoço. As tarefas foram avaliadas após várias visitas às empresas, sendo todas as atividades repetitivas gravadas em vídeo. Dados a respeito das características individuais dos trabalhadores e das características psicossociais do trabalho foram coletados por questionários. A dor no ombro e no pescoço foi avaliada por exame físico realizado por três grupos de médicos. Nos resultados, o autor encontrou prevalência maior de queixas no ombro e no pescoço entre os trabalhadores com trabalho monótono $(7,0 \%)$, quando comparados aos trabalhadores sem atividade monótona (3,8\%). A combinação entre repetitividade e força aumentou o risco para dor no ombro e no pescoço (Razão de prevalência 2,3; IC, 1,4-4.0) ${ }^{12}$.

Com o objetivo de investigar associações e interações entre trabalho e dor no ombro e no pescoço, Vasseljen e Westgaard (1995) conduziram estudo caso-controle entre 39 norueguesas trabalhadoras de escritório. Os casos se constituíam de 15 indivíduos que apresentavam dor no ombro e no pescoço de origem ocupacional; os controles, 24 , eram todas do mesmo setor, com a mesma idade. Foi realizada eletroneuromiografia de superfície no músculo trapézio dos participantes e outros dados foram coletados por meio de questionários. Os autores notaram associação estatisticamente significante entre a percepção de trabalhar sob tensão e atividade muscular aumentada do trapézio 
medida por eletroneuromiografia. Entre as trabalhadoras com queixas músculo-esqueléticas, a eletroneuromiografia de atividade no repouso e durante o trabalho apresentava padrões alterados quando comparada ao grupo controle. O estudo, porém, não foi capaz de explicar a alteração. Levantou-se possível relação com as características individuais das trabalhadoras ${ }^{13}$.

Punnet et al. (2000) em estudo do tipo caso-controle, no Estado de Michigan, identificaram os casos (79) de dor no ombro nos últimos 90 dias, em prontuários médicos de uma empresa automobilística, e os controles (124) foram selecionados aleatoriamente entre os trabalhadores do setor administrativo da empresa. O tipo de trabalho executado pelos trabalhadores com queixa foi gravado em vídeo e posteriormente analisado. Verificou-se que os casos passavam mais tempo com o ombro em flexão do que os controles. O risco de desenvolver problemas foi duas vezes maior nos trabalhadores que fletiam ou abduziam o ombro (Odds Ratio, 4,0; IC, 1,7-9,4). Não houve diferenças nas queixas quando se carregava peso inferior a 44, 5 Newtons ${ }^{14}$.

Visando avaliar o efeito da exposição no ambiente de trabalho, a curto e a longo prazo, para dores de ombro e de pescoço, Fredriksson et al. (2002), na Suécia, realizaram estudo tipo caso-controle, em 1.587 pessoas. Os casos foram definidos como aqueles que apresentavam dor na região do pescoço e do ombro, nos últimos seis meses, e que haviam procurado auxílio médico. Os controles foram selecionados aleatoriamente. Os dados relacionados à sintomatologia e ao tipo de trabalho desempenhado foram colhidos por meio de questionários. Os pacientes também foram examinados por seis médicos e classificados em quatro grupos: sem sinais objetivos de doença cervical ou do ombro; com sinais de tensão cervical; com sinais de tendinite do ombro; e com sinais de artrose cervical. Houve prevalência de tendinite do ombro entre os casos de $31 \%$, para os homens, e 43\%, para as mulheres. Foi encontrada relação entre vibração e carregar peso e dor no ombro ${ }^{15}$.
Fredriksson et al. (1999) conduziram estudo prospectivo que teve início no ano de 1969, em 2.500 pessoas, que viviam em Estocolmo, com idade variando dos 18 aos 65 anos, e que foram avaliadas por meio de exame clínico e entrevista, com coleta de dados sobre as condições do trabalho. Em 1993, foram então reavaliadas 484 pessoas, com idade abaixo de 59 anos, e que não tinham queixas músculo-esqueléticas no ano de 1969, por meio de questionário que coletava dados sobre problemas na região cervical, dos ombros, cotovelo, punho e mãos. Foram encontradas correlações fortes entre sintomas no ombro e quantidade de horas-extras realizadas, entre as mulheres, e forte correlação entre períodos de repouso insatisfatórios e dor no ombro, entre os homens ${ }^{16}$.

A ocorrência e a persistência de dor no ombro e no pescoço foram avaliadas entre operadoras de máquina de costura por Kaergaard e Andersen (2000), que conduziram estudo prospectivo por dois anos na Dinamarca. Cento e setenta e oito operadoras assintomáticas, no ano de 1994, foram acompanhadas prospectivamente por dois anos como controle e foram selecionadas 357 mulheres expostas a trabalho pouco repetitivo. Durante o estudo, as trabalhadoras preencheram três questionários com informações a respeito da carga do trabalho, fatores psicossociais e sintomas músculoesqueléticos. Quando havia queixa de dor músculo-esquelética, as trabalhadoras eram submetidas a exame do ombro. Houve maior prevalência de tendinite do manguito rotador nas operadoras de máquina de costura, quando comparadas ao grupo controle (Odds Ratio, 2,26; IC, 1.1-5,9). A prevalência de tendinite do manguito rotador aumentou com o tempo de emprego ${ }^{17}$.

Com o objetivo de avaliar o efeito do trabalho e dos fatores individuais na incidência e na persistência de dor no ombro, entre 3312 trabalhadores, o estudo prospectivo de Miranda et al. (2001) foi realizado durante quatro anos. Os trabalhadores florestais finlandeses sem queixa músculo-esquelética foram acompanhados prospectivamente até 1995. A cada ano um questionário foi envia- 
do aos participantes. Obteve-se incidência anual de dor no ombro de $14 \%$. Houve risco aumentado de dor no ombro com a idade e nos pacientes com maior esforço no trabalho (Odds Ratio, 2,4; IC, 1,7-3,4) e naqueles com estresse mental maior (Odds Ratio 2,1; IC, $1,3-3,5)^{18}$.

Com o intuito de avaliar o efeito da sobrecarga física e psicossocial sobre o ombro e o pescoço, Feveile et al. (2002) realizaram estudo prospectivo, durante cinco anos. Foram acompanhados 3.390 trabalhadores dinamarqueses, no período de 1990 a 1995 . A todos os participantes foi enviado um questionário, em 1990, e outro em 1995. Entre os homens, torção ou inclinação do pescoço e ombro durante o trabalho, assim como baixo suporte social foram relacionados ao aparecimento de dor. Entre as mulheres, somente o hábito de fumar foi associado ao desenvolvimento de sintomas no ombro e no pescoço ${ }^{19}$.

Estudo prospectivo, realizado por Hoozemans et al. (2002), com duração de um ano, avaliou possíveis associações entre a realização de tarefas de empurrar e puxar objetos com sintomas no ombro. A população de 459 trabalhadores, homens e mulheres, de várias empresas holandesas, estava envolvida em tarefas de empurrar e puxar objetos. O grupo controle foi formado pelos trabalhadores do setor administrativo ou gerentes das empresas pesquisadas. O estudo evidenciou prevalência de dor no ombro significativamente maior em trabalhadores que exerciam atividades de empurrar e puxar objetos pesados (RR, 3,95; IC, 5,6-9,96), tendo sido encontrado um efeito clássico do tipo dose/resposta ${ }^{20}$.

Cassou et al. (2002) relataram os resultados de um estudo prospectivo, de cinco anos de duração em 18.869 trabalhadores de cinco regiões da França. Os dados foram colhidos, inicialmente, em 1990, por meio de dois questionários, que continham perguntas a respeito de dor no ombro e no pescoço e das atividades desenvolvidas pelos trabalhadores. Todos os indivíduos foram submetidos a exame físico músculo-esquelético no início do estudo. Os dados foram novamente coletados, da mesma maneira, em 1995. Os autores encontraram que os homens exerceram atividades em posturas viciosas e, as mulheres, tarefas com maior repetitividade. Entre os homens, a prevalência de dor no ombro e no pescoço aumentou de 7,8\%, em 1990, para 9,5\%, em 1995; e, entre as mulheres, a prevalência aumentou de 14,8\%, em 1990, para $17,6 \%$, em 1995. Com o aumento da idade, houve incidência maior de dor, tanto em homens quanto em mulheres. Para os homens, houve relação estatisticamente significativa entre tarefas repetitivas, posições viciosas e alta demanda física no trabalho, com dor no ombro e no pescoço. Para as mulheres, houve relação estatisticamente significativa entre tarefas repetitivas, posições viciosas, movimentos precisos, alta demanda física e pouco controle do trabalho com dor no ombro e pescoço. Para ambos os sexos, os fatores ocupacionais aumentaram a incidência de dor no ombro e no pescoço, independentemente da idade ${ }^{21}$.

Na Dinamarca foram acompanhadas 12 mulheres, com finalidade de estudar o aparecimento de dor no ombro e no pescoço em trabalhadoras recém admitidas em um frigorífico. Todas estavam assintomáticas à admissão e foram submetidas a exame físico para a avaliação de pontos dolorosos no pescoço e no ombro. As tarefas desenvolvidas foram observadas em situação real de trabalho. As trabalhadoras foram, então, reavaliadas após seis meses. Os autores identificaram interação significante entre duração do emprego e queixas de dor no ombro e no pescoço. Houve diferença estatisticamente significante entre a posição do braço, no início da tarefa e a presença ou não de queixas álgicas. Na conclusão, os autores chamam a atenção para a diferença da postura adotada no início da realização da tarefa entre os dois grupos, o que pode significar um processo de adaptação ao trabalho com o intuito de evitar posições viciosas. Assim, ao longo de seis meses, as trabalhadoras desenvolveram estratégias sensório-motoras, realizando a tarefa de forma mais adequada e com diminuição na amplitude dos movimentos do braço (Madeleine et al, 2003) ${ }^{22}$. 
Em relação às pesquisas experimentais, nesta revisão foram encontrados quatro estudos suecos que tentaram, em laboratório, simular as condições encontradas pelos trabalhadores no ambiente de trabalho, conforme resultados relatados a seguir.

Em voluntários, Sporrong et al. (1998) observaram aumento da atividade muscular, medida por eletroneuromiografia, nas tarefas que exigiam precisão manual. Dez pessoas hígidas participaram do experimento. A atividade mioelétrica foi medida em seis músculos do ombro: deltóide, supra e infra-espinhoso, trapézio, elevador da escápula e rombóide maior. A atividade era medida com os voluntários sentados e o ombro executando atividades de precisão entre $30^{\circ}, 45^{\circ}, 60^{\circ}$ e $90^{\circ}$ deflexão e o cotovelo nas posições de $0^{\circ}, 60^{\circ} \mathrm{e} 90^{\circ}$. Os autores verificaram aumento na atividade do supra-espinhoso em todas as posições do ombro. Aatividade do infra-espinhoso também aumentou em todas as posições. Na conclusão, os autores relatam que, para executar tarefas de precisão, existe a necessidade de estabilização do ombro, obtidas pelo aumento da atividade do supra e do infra-espinhoso, aumentando o estresse em tais músculos ${ }^{23}$.

Ainda na Suécia, com o objetivo de avaliar o impacto de atividades de repetitividade sobre a propriocepção do ombro, Björklund et al. (2000) conduziram estudo experimental que contou com a participação de 18 indivíduos sadios. O experimento consistia na medida da propriocepção, antes e depois de realizar tarefa repetitiva para o ombro. $\mathrm{O}$ participante ficava sentado em uma cadeira com uma armação motorizada para o braço, mantendo os olhos fechados. Da posição inicial, de $45^{\circ}$ no plano sagital, movimentos de $15^{\circ}$ a $30^{\circ}$ de adução e $60^{\circ}$ a $75^{\circ}$ de abdução eram realizados. Ao comando de "parar", o participante parava os movimentos e a armação para o braço era travada por cinco segundos. Então o participante, com os olhos fechados, teria que retornar o braço à sua posição inicial. Para realizar tarefas repetitivas, o participante sentava- diante de uma mesa e realizava a tarefa de empurrar e puxar um pistão e pressionar um botão. Os autores encontraram diminui- ção da propriocepção quando comparando os resultados pré e pós-fadiga. As mulheres apresentaram diminuição mais importante do que os homens. Na conclusão, os autores relatam que tal diminuição na propriocepção, induzida pela atividade, pode ser o fator inicial na ocorrência de doenças músculo-esqueléticas de origem ocupacional. A alteração da propriocepção, por ser maior entre as mulheres, justificaria maior incidência de doenças músculo-esqueléticas de origem ocupacional nesse gênero ${ }^{24}$.

Para avaliar o efeito da posição do ombro, com e sem carga sobre a pressão intramuscular dos músculos supra e infraespinhoso, Palmerud et al. (2000) realizaram estudo experimental. Foram medidas as pressões intramusculares nos músculos supra $\mathrm{e}$ infra-espinhoso, em dez voluntários, em diversas posições do ombro, com ou sem carga sobre a mão. Notou-se a tendência à elevação da pressão intramuscular dos músculos supra e infra-espinhoso, quando se partia da posição de repouso do ombro $\left(0^{\circ} \mathrm{de}\right.$ elevação e abdução) para a posição de $90^{\circ} \mathrm{de}$ elevação anterior, posição esta em que a pressão era maior. Quando se aplicava carga na mão dos pacientes, o aumento na pressão intramuscular do supra-espinhoso não foi estatisticamente significativo, porém o aumento da pressão intramuscular do infraespinhoso foi estatisticamente significante. A partir de experimentos, os autores concluíram que fatores como posição do ombro e carga sobre o membro superior influenciam o aumento da pressão intramuscular do supra e do infra-espinhoso. Elevação prolongada do ombro então poderia levar a alteração do fluxo sangüíneo nos músculos supra e infra-espinhoso devido ao aumento na pressão intramuscular dos mesmos, favorecendo o cansaço muscular no ombro, assim como ruptura dos tendões do manguito rotador ${ }^{25}$.

Stenlund et al. (2002) realizaram estudo experimental entre pintores de parede (40) com o intuito de descobrir possíveis correlações entre carga nos ombros e desenvolvimento de dor. Enquanto pintavam uma tábua quadrada, situada a 2,5 metros de altu- 
ra, um sistema de câmeras de vídeo gravava os movimentos. Um questionário também foi aplicado aos pintores para avaliar os sintomas no pescoço, no ombro, no cotovelo, no punho e no dorso. Ao término das gravações, foi possível identificar três tipos de técnicas para pintar o teto. Encontrou-se associação estatisticamente significante entre $o$ tipo de técnica utilizada pelo pintor e a dor no ombro. Os pintores que usavam a técnica de puxar tinham menos sintomas no ombro que os demais. Concluiu-se que o tipo de técnica empregada pelo trabalhador pode ter efeito sobre a sintomatologia músculo-esquelética ${ }^{26}$.

\section{Discussão}

Os vinte artigos apresentam-se heterogêneos quanto à avaliação da exposição, ao tipo de diagnóstico e à metodologia utilizada na condução da pesquisa. Encontrou-se a avaliação da dor por meio de questionários e de exame físico

Quanto ao diagnóstico, para alguns autores foi suficiente o dado positivo de dor no ombro como indicativo de distúrbios; para outros foi necessária a presença de lesão do manguito rotador. Vê-se, dessa forma, a impossibilidade de comparação entre os artigos avaliados.

No que se refere à exposição ocupacional, as investigações que resultaram nos artigos desta revisão utilizaram, como instrumentos, questionários, observações diretas ou filmagens do ambiente de trabalho. A diversidade de avaliação de exposição tam- bém dificulta a comparação entre os resultados apresentados nos diferentes artigos. Além disso, os instrumentos variam quanto ao poder de elucidação da carga física do trabalho, que pode ser mais ou menos valorizada a depender da presença ou não de queixas entre os trabalhadores do estudo.

Os artigos encontrados não definem "os casos" de maneira clara e precisa, e nem sempre a metodologia utilizada é coerente com os objetivos expostos. Esse problema pode ser devido ao caráter multifatorial do evento clínico, que pode se apresentar em mais de uma região do corpo e, ainda, de intensidade também variada.

\section{Conclusão}

Da análise dos estudos acima, pode-se afirmar que os distúrbios do ombro são influenciados por fatores biomecânicos relacionados ao trabalho, como flexão ou abdução dos ombros por tempo prolongado, vibrações, postura estática ou com carga no membro superior. Uma relação do tipo dose/resposta entre sobrecarga e doenças do ombro foi encontrada nos trabalhos. Os resultados dos estudos evidenciam associação entre os distúrbios do ombro e os fatores psicossociais como estresse, longas jornadas de trabalho, período de descanso insatisfatório. Pode-se sugerir futuros estudos e linhas investigatórias que desenvolvam padronizações dos métodos de avaliação da exposição e do efeito musculo esquelético, visando oferecer meios mais seguros para a proposição de medidas preventivas.

\section{Referências}

1. Rockwood CAJ, Matsen III FA. The shoulder. Philadelphia: W.B. Saunders Company, 1988.

2. Ministério da Previdência Social. Estatísticas de acidente do trabalho. Disponível em http:// previdência social.gov.br. Acesso em 26 set. 2004.

3. Ministério da Saúde do Brasil. Representação no Brasil da OPAS/OMS. Doenças Relacionadas ao Trabalho. Manual de procedimentos para os serviços de saúde. Brasília, 2001.
4. Wint DAM et al. Occupational risk factors for shoulder pain: a systematic review. Occup Environ Med 2000; 57: $433-42$.

5. Silverstein B, Welp E, Nelson N, Kalat BA. Claims incidence of work-related disorders of the upper extremities: Washington State, 1987 through 1995. Am. J Public Health 1988; 88: 1827-33. 
6. European Agency for Safety and Healthy at Work. Repetitive strain injuries in the member states of the European Union: the results of an information request. Bruxelas, 2000.

7. National Institute of Occupational Safety and Health [NIOSH]. USA. Cumulative trauma disorders in the workplace. 1997. Disponível em http://www.cdc.gov/ niosh/nrworg.html. Acesso em 30 ago. 2003.

8. Stenlund B, Goldie I, Hagberg M, Hogstedt C. Shoulder tendinitis and its relation to heavy manual work and exposure to vibration. Scand J Environ Health 1993; 19: 43-9.

9. Mäkela M, Heliovaara M, Sainio $P$, Knekt $P$, Impivaara O, Aromaa A. Shoulder joint impairment among Finns aged 30 years or over: prevalence, risk factors and comorbidity. Rheumatology 1999; 38: 656-62.

10. Frost P, Andersen JH. Shoulder impingement syndrome in relation to shoulder intensive work. Occup Environ Med. 1999: 56: 494-8.

11. Pope MH, Goh KL, Magnusson ML. Spine ergonomics. Annu Rev Biomed Eng 2002; 4: 49-68.

12. Andersen JH. et al. Physical, psychosocial, and individual risk factors for neck/shoulder pain with pressure tenderness in the muscles among workers performing monotonous, repetitive work. Spine 2002; 27: $660-7$.

13. Vasseljen O, Westgaard RH. Can stress-related shoulder and neck pain develop independently of muscle activity? Pain 1995; 64: 221-30.

14. Punnett L; Lawrence JF, Keyserling WM, Herrin GD, Chaffin DB. Shoulder disorders and postural stress in automobile assembly work. Scand J Work Environ Health 2000; 26: 283-91.

15. Fredriksson $\mathrm{K}$ et al. Work environment and neck and shoulder pain: the influence of exposure time. Results from a population based case-control study. Occup Environ Med 2002; 59: 182-8.

16. Fredriksson K. et al. Risk factors for neck and upper limb disorders: results from 24 years of follow-up. Occup Environ Med 1999; 56: 59-66.

17. Kaergaard A, Andersen JH. Musculoskeletal disorders of the neck and shoulders in female sewing machine operators: prevalence, incidence, and prognosis. Occup Environ Med 2000; 57: 528-34.
18. Miranda H, Viikari-Juntura E, Martikainen R, Takala EP, Riihimaki H. A prospective study of work related factors and physical exercise as predictors of shoulder pain. Occup Environ Med 2001; 58: 528-34.

19. Feveile H, Jensen C, Burr H. Risk factors for neckshoulder and wrist-hand symptoms in a 5-year followup study of 3,990 employees in Denmark. Int. Arch. Occup Environ Health 2002; 75: 243-51.

20. Hoozemans MJM, Beek AJ, Frings-Dresen MHW, Woude LHV, Dijk FJH. Low-back and shoulder complaints among workers with pushing and pulling tasks. Scand J Work Environ Health 2002; 28: 293-303.

21. Cassou B, Derriennic F, Monfort C, Norton J, Touranchet A. Chronic neck and shoulder pain, age, and working conditions: longitudinal results from a large random sample in France. Occup. Environ Med 2002: 59; 537-44.

22. Madeleine P, Lundager B, Voigt M, Arendt-Nielsen L. The effects of neck-shoulder pain development on sensory-motor interactions among female workers in poultry and fish industries. A prospective study. Int Arch Occup Environ Health 2003; 76: 39-49.

23. Sporrong H, Palmerud G, Kadefors R, Herberts P. The effect of light manual precision work on shoulder muscles- an EMG analysis. Jour Electromyografy and Kinesiology 1998; 8: 177-84.

24. Bjorklund M, Crenshaw AG, Djupsojobacka M, Johansson $\mathrm{H}$. Position sense acuity is diminished following repetitive low-intensity work to fatigue in a simulated occupational setting. Eur J Appl Phyliol 2000; 81: 361-7.

25. Palmerud G, Forsman M, Sporrong H, Herberts P, Kadefors R. Intramuscular pressure of the infra- and supraspinatus muscles in relation to hand load and arm posture. Eur J Appl Physiol 2000; 83: 223-30.

26. Stenlund B, Lindbeck L, Karlsson D. Significance of house painters' work techniques on shoulder muscle strain during overhead work. Ergonomics 2002; 45: 455-68.

recebido em: 18/01/05 versão final reapresentada em: 06/05/05 aprovado em: 11/05/05 